\title{
Two-level QoS-aware frame-based downlink resources allocation for RT/NRT services fairness in LTE networks
}

\begin{abstract}
In LTE, bandwidth resources allocation became a huge burden to be handled, as both of RT and NRT traffic should be processed fairly to satisfy their diverse QoS requirements. To tackle this issue in particular, in this work, we propose a fairness-based resources allocation method named Frame-based Game Theory (FGT), which can be flexibly implemented as an upper level in LTE downlink MAC layer; before the PRBs allocation function. Basically, FGT aim is to allow involved classes with different QoS requirements to fairly gain a part of the available channel resources to transmit their flows. Adhere, on every LTE-frame, a cooperative game scenario is designed. Wherein, Shapley formula is used to distribute the available amount of data within RT and NRT traffic classes. Then, conforming to these assigned resources portions to each traffic class, PRBs allocation process is triggered every TTI for the selected flows. In this work, we adopted some of the recent PRBs allocation methods as a lower level scheduler in order to illustrate a complete idea of MAC layer scheduling functions, and also to effectively evaluate FGT performance. Simulation results on various scenarios show that, fairness index for flows from different classes is increased when FGT is considered. The results also conveyed outperforming QoS indices for FGT on RT and NRT service in terms of throughput, PLR, as well as cell spectrum efficiency.
\end{abstract}

Keyword: Downlink packet scheduling; QoS; Game theory; Shapley value; Fairness; Throughput 